\title{
Stackable acoustic holograms
}

Cite as: Appl. Phys. Lett. 116, 261901 (2020); https://doi.org/10.1063/5.0009829

Submitted: 03 April 2020 . Accepted: 13 June 2020 . Published Online: 29 June 2020

Michael D. Brown (D), Ben T. Cox (D), and Bradley E. Treeby (D)

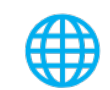

\section{Lock-in Amplifiers up to $600 \mathrm{MHz}$

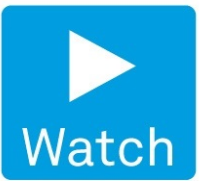

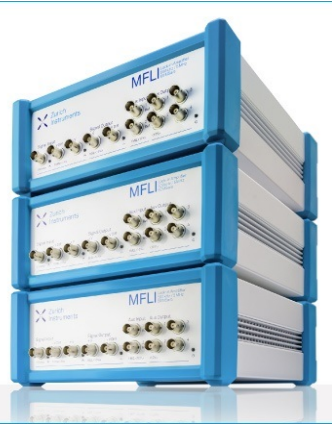




\title{
Stackable acoustic holograms
}

\author{
Cite as: Appl. Phys. Lett. 116, 261901 (2020); doi: 10.1063/5.0009829 \\ Submitted: 3 April 2020 - Accepted: 13 June 2020 • \\ Published Online: 29 June 2020
}
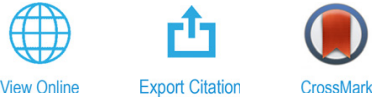

Michael D. Brown, ${ }^{\text {a) }}$ (D) Ben T. Cox, (iD) and Bradley E. Treeby iD

\begin{abstract}
AFFILIATIONS
Department of Medical Physics and Biomedical Engineering, University College London, Gower St., London WC1E 6BT, United Kingdom
\end{abstract}

${ }^{a)}$ Author to whom correspondence should be addressed: Michael.brown.13@ucl.ac.uk

\begin{abstract}
Acoustic holograms can be used to form complex distributions of pressure in 3D at MHz frequencies from simple inexpensive ultrasound sources. The generation of such fields is vital to a diverse range of applications in physical acoustics. However, at present, the application of acoustic holograms is severely hindered by the static nature of the resulting fields. In this work, it is shown that by intentionally reducing the diffraction efficiency of each hologram, it is possible to create stackable acoustic holograms that can be repositioned to reconfigure the combined acoustic field. An experimental test-case consisting of two holograms, each designed to generate a distinct distribution of acoustic foci, is used to demonstrate the feasibility of this approach. Field scans taken for four different positions of the two holograms confirm that the individual patterns for each hologram can be arbitrary translated relative to one another. This allows for the generation of a much greater range of fields from a single transducer than could be created using a single hologram.
\end{abstract}

Published under license by AIP Publishing. https://doi.org/10.1063/5.0009829

The ability to precisely control acoustic fields in $3 \mathrm{D}$ is essential to applications in physical acoustics ranging from particle manipulation ${ }^{1,2}$ and haptics ${ }^{3,4}$ to ultrasound therapy, ${ }^{5,6}$ neuro-stimulation, ${ }^{7}$ energy transfer, ${ }^{9}$ and imaging. ${ }^{10}$ Such control is conventionally achieved using large arrays of separate elements. ${ }^{8}$ However, both the fabrication and driving of such arrays are a challenge, and they scale poorly with increasing frequency and aperture size due to the large number of elements required to fully sample the aperture. Acoustic holograms are an alternative method for generating arbitrary distributions of pressure in three dimensions. These are phase plates, often 3D printed, that can be used to map the continuous wave output of a single element transducer (or array) at a specific design frequency onto a target phase distribution using variations in thickness and sound-speed contrast. ${ }^{11-13}$ The phase distribution onto which the field is mapped is designed to diffract to form a desired distribution of acoustic pressure.

Acoustic holograms can be fabricated at low cost $(\sim £ 5)$, scale with no additional complexity to larger apertures, and, with the resolution of current 3D printing technology, can be fabricated for frequencies up to $\sim 7.5 \mathrm{MHz}$. Compared with conventional arrays, they offer higher precision control over the phase distribution; ${ }^{14}$ however, they are passive devices. Each hologram is fixed and designed to form one particular field for a particular driving frequency.

To circumvent this limitation, several approaches have been reported. One option is to design a multi-frequency hologram for which distinct patterns are encoded onto different driving frequencies. ${ }^{15}$ However, the number of patterns is limited by both crosstalk and the operational bandwidth of the transducer. Alternatively, Lalonde and Hunt ${ }^{16}$ demonstrated that the fixed pattern of an acoustic hologram could have either its focal depth changed or its size altered by adjusting the driving frequency from the design frequency. These changes occur due to the frequency variation of the phase offsets introduced by propagation from the hologram surface to the target depth. However, the effect was only explored for a relatively narrow (50\%) bandwidth around the design frequency. Outside this range the change in the resulting field might be expected to become more complex due to large changes in the phase offsets introduced by the hologram. Scaling and steering of a fixed pattern can also be achieved by using an acoustic hologram in conjunction with a phased array as opposed to a single element transducer. For a fully sampled array, adding a hologram provides no benefit. However, for an undersampled array with fewer elements/channels, a hologram can be used to generate a complex pattern that could not be formed using the array alone. The pattern can then be steered by applying different delays to the underlying elements. ${ }^{14,17}$ For this approach, the steering is limited by grating lobes related to the spacing of the array elements.

In this paper, we introduce an alternative approach that enables the generation of two or more distinct patterns of pressure from a single element transducer by using two or more stacked holograms. The relative position or angle between each pattern can additionally be freely reconfigured by changing the position of or rotating its respective 
hologram, allowing the combined pattern to be flexibly changed. The essential idea is to intentionally decrease the modulation depth of each hologram, thereby coupling more energy into the un-diffracted zeroth order. Each hologram interacts with this un-diffracted portion of the wave to generate its respective target pattern.

The phase profile of a transmission hologram designed to generate a single focus along the transducer axis at a depth $d$ at a frequency $f_{0}$ from a planar transducer can be seen in Fig. 1. This has an axisymmetric profile and it has been shown by Moreno et al. ${ }^{18}$ that, for a normally incident plane wave, the field $t(r)$ transmitted by this hologram can be decomposed into a set of waves that focus on-axis at different depths (diffraction orders) described by

$$
t(r)=\sum_{j=-\infty}^{+\infty} C_{j} \exp \left(i \frac{\pi j}{\lambda_{0} d} r^{2}\right) .
$$

Here $j$ is the diffraction order, $C_{j}$ is the amplitude of each diffraction order, $\lambda_{0}$ is the wavelength in the coupled medium, $i$ is the unit imaginary number, and $r$ is the radial distance from the center of the hologram. The coefficients $C_{j}$ are given by

$$
C_{j}^{2}=\operatorname{sinc}^{2}(\alpha-j),
$$

where $\alpha$ is a design parameter which is proportional to the modulation depth of the hologram $\Delta \phi_{m}$ (the maximum phase offset it introduces). $C_{j}^{2}$ represents the fraction of the incident energy in each diffraction order. The modulation depth $\Delta \phi_{m}$ is determined by the sound speed in the hologram $c_{h}$ and the coupled medium $c_{m}$, the maximum change in thickness $h_{m}$, and the frequency via

$$
\alpha=\frac{\Delta \phi_{m}}{2 \pi}=f_{0}\left(\frac{1}{c_{m}}-\frac{1}{c_{h}}\right) h_{m} .
$$

From Eqs. (2) and (3), it can be seen that by varying the modulation depth, the distribution of the field transmitted by the hologram into different diffraction orders can be controlled. Figure 2(a) shows this variation for $j=0$ to $j=3$ and $\Delta \phi_{m}$ from 0 to $10 \pi$. The effect that this has on the transmitted field for the hologram in Fig. 1 for four values of $\Delta \phi_{m}$ can be seen in Figs. 2(b)-2(e). For $\Delta \phi_{m}=2 \pi$, all the energy is, in principle, coupled into the first diffraction order, generating a single focus at $d$ [Fig. 2(d)]. Alternatively, increasing $\Delta \phi_{m}$ to $3 \pi$ results in $40 \%$ of the energy in both the first and second diffraction orders. This creates two foci one at the original target depth and one

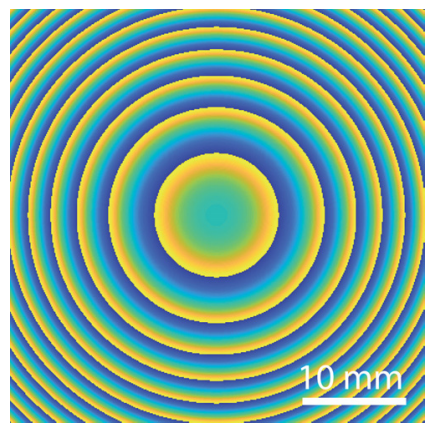

FIG. 1. Phase profile for hologram to generate a single focus in water for $\mathrm{d}=2.5 \mathrm{~cm}$ and $f_{0}=2.5 \mathrm{MHz}$ closer to the hologram [Fig. 2(e)]. Conversely, decreasing $\Delta \phi_{m}$ below $2 \pi$ results in energy being increasingly coupled into the zeroth order [Fig. 2(c)]. This zeroth order, which can be seen in Fig. 2(b), represents the portion of the wave that is unchanged by propagation through the hologram and is identical to the field in the absence of the hologram. This final case where a large fraction of the incident field is unaffected by propagation through the hologram is what this work aims to exploit.

Assuming two holograms $h_{1}$ and $h_{2}$ with target focal depths of $d_{1}$ and $d_{2}$ and modulations depths of $\pi$ are directly stacked, ignoring the finite thickness of both holograms, the transmitted field will be given by $t_{h_{1}}(r) t_{h_{2}}(r)$. It can be seen from Fig. 2(a) that both holograms will have their energy evenly divided principally into the first and zeroth diffraction orders. As a result, the transmitted field will have four main components

$$
\begin{gathered}
p_{00}=C_{0}^{h_{1}} C_{0}^{h_{2}}, \\
p_{10}=C_{1}^{h_{1}} C_{0}^{h_{2}} \exp \left(i \frac{\pi}{\lambda_{0} d_{1}} r^{2}\right), \\
p_{01}=C_{0}^{h_{1}} C_{1}^{h_{2}} \exp \left(i \frac{\pi}{\lambda_{0} d_{2}} r^{2}\right), \\
p_{11}=C_{1}^{h_{1}} C_{1}^{h_{2}} \exp \left(i \frac{\pi r^{2}}{\lambda_{0}}\left(\frac{1}{d_{1}}+\frac{1}{d_{2}}\right)\right) .
\end{gathered}
$$

The first $p_{00}$ is the incident field. The second and third terms $p_{10}$ and $p_{01}$ are waves that focus at $d_{1}$ and $d_{2}$, respectively. The fourth term $p_{11}$ is a doubly diffracted wave that focuses at some depth $d_{11}$ that is less than $d_{1}$ and $d_{2}$. Equation (4) shows that both the original target foci are still formed despite the holograms being stacked. Additionally, the $p_{01}$ and $p_{10}$ terms are independent of the structure or orientation of the other hologram aside from its modulation depth. In principle, if $p_{11}, p_{00}$, and other higher order terms can be neglected at the focal depth, then this should allow the two holograms to be repositioned without perturbing each other's field. Here, we experimentally validate this idea for holograms designed to generate complex multi-focal patterns and demonstrate that the combined field can be freely reconfigured simply by changing the orientation of the two holograms.

First, two phase holograms $\phi_{1}(x, y)$ and $\phi_{2}(x, y)$ were independently calculated. The target patterns for the two holograms can be seen in Figs. 3(a) and 3(b). Both were designed for a $2.54 \mathrm{~cm}$ diameter 2.25 MHz piezoelectric transducer (Olympus, Japan) for a design frequency of $3 \mathrm{MHz}$. The target pattern depth for the first hologram was $3.2 \mathrm{~cm}$ and for the second, it was $3 \mathrm{~cm}$. This offset was introduced to compensate for the fabricated thickness of both holograms to ensure the focal planes will overlap. Each hologram was calculated using an iterative angular spectrum approach. ${ }^{12,19}$ This works by propagating the acoustic field repeatedly between the target plane and the transducer surface using the angular spectrum method. In each plane, the amplitude of the field is replaced and the phase is preserved. In the transducer plane, the amplitude is set to the measured transducer surface pressure while in the target plane the amplitude is set to the target pressure distribution. This process is iterated until it converges to a phase distribution $\phi(x, y)$ for the transducer surface that approximately generates the target pattern. For this work, however, the position of the second hologram $\phi_{2}(x, y)$ relative to the transducer surface is intended to vary between different measurements. Therefore, in 
(b)

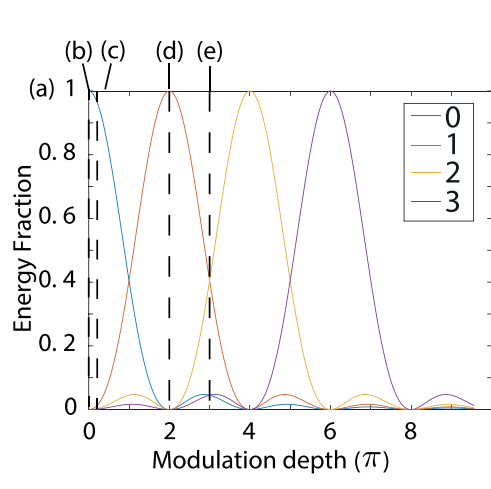

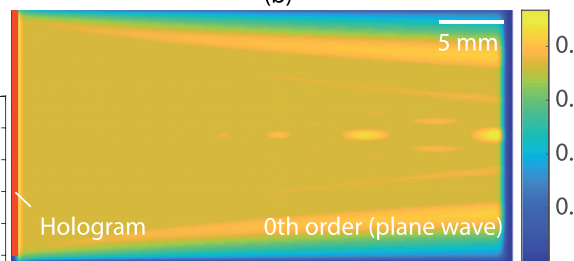

(d)

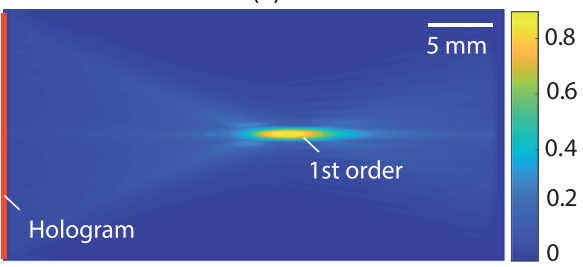

(c)

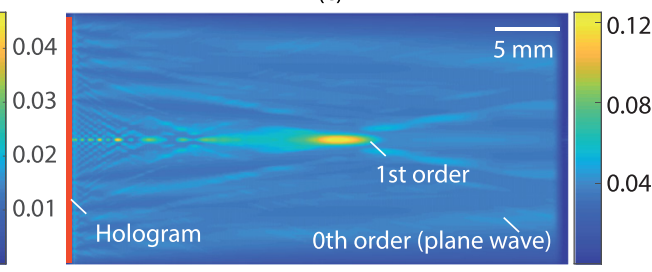

(e)

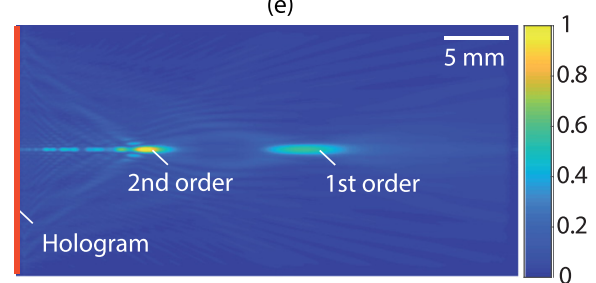

FIG. 2. (a) Fraction of energy distributed by the hologram into each order as a function of modulation depth calculated using Eqs. (2) and (3). (b)-(e) Acoustic field generated by single focus hologram for holograms with modulation depths (b) $0 \pi$, (c) $\frac{\pi}{5}$, (d) $2 \pi$, and (e) $3 \pi$. The colorbar for (b)-(e) represents the peak pressure normalized over the four different plots.

calculating the second hologram, rather than using the transducer surface pressure as the amplitude constraint for the transducer plane, the amplitude was instead set to a uniform value over a $5 \times 5 \mathrm{~cm}$ area.

After calculation, both phase distributions were then converted into hologram surface profiles $h_{1}$ and $h_{2}$ that both had maximum modulation depths $\Delta \phi_{m}$ of $\pi$ using

$$
h(x, y)=\frac{\pi-\Delta \phi(x, y)}{2 \pi f_{0}\left(\frac{1}{c_{m}}-\frac{1}{c_{h}}\right)} .
$$

In previous works, ${ }^{12,15}$ holograms have been fabricated from a single solid material with water as the coupled medium. However, for stacking two holograms, this could lead to air being trapped between the holograms due to the rough surface profile. To avoid this, the first hologram $h_{1}$ was fabricated using two solid 3D printing materials to provide a flat surface for the second hologram to attach to. These materials, tangoBlack $\left(c_{h}\right)$ and veroClear $\left(c_{m}\right)$, have sound speeds of $1937 \mathrm{~ms}^{-1}$ and $2495 \mathrm{~ms}^{-1}$, respectively. The second hologram $h_{2}$ was fabricated from veroClear with water as the coupled medium $c_{m}$. A surrounding buffer was added to both holograms, so that they could be attached and re-positioned. Four clearance holes were added to $h_{1}$, while 16 were added to $h_{2}$ allowing them to be attached to each other in four distinct positions. To attach $h_{1}$ to the transducer, a ring was

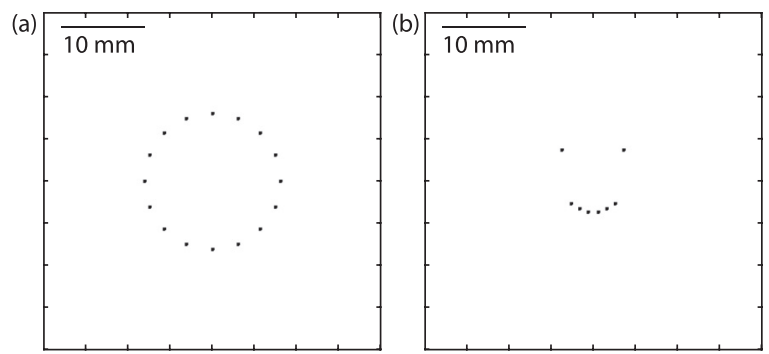

FIG. 3. (a) Target pattern for hologram 1. (b) Target pattern for hologram 2. added to the rear surface. Both holograms were fabricated on a highresolution multi-material polyjet printer (Objet350 Connex, Stratasys, Eden Prairie, MN, USA). The resulting holograms can be seen in Figs. 4(a) and 4(b).

Measurements of the generated acoustic field were carried out in a $40 \times 40 \times 60 \mathrm{~cm}^{3}$ test tank with a two-axis computer controlled positioning system (Precision Acoustics, Dorchester, UK) using a calibrated $0.2 \mathrm{~mm}$ needle hydrophone (Precision Acoustics, Dorchester). The two holograms were attached to each other and then to the transducer surface. The transducer was driven using a signal generator (33522A, Agilent Technologies, Santa Clara, CA, USA) connected via a $75 \mathrm{~W}$ power amplifier (A075, E\&I, Rochester, NY, USA). The driving signal was a $\sim 40 \mathrm{Vpp} 50$ cycle tone burst at $3 \mathrm{MHz}$. The number of cycles was set to ensure that the signal covered the minimum and maximum travel times from the hologram to the measurement plane (approximately $40 \mathrm{~mm}$ from the front surface). The field was recorded over a $40 \times 40 \mathrm{~mm}^{2}$ area, centered on the middle of the hologram with a step size of $0.25 \mathrm{~mm}$. Signals were recorded on an InfiniiVision DSOX3024A oscilloscope (Keysight, California, USA). The measurements were then back-propagated to the target depth using the
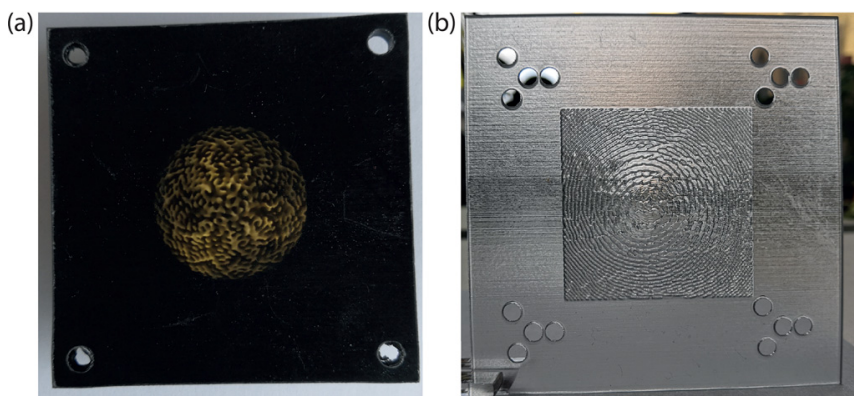

FIG. 4. (a) Hologram 1 fabricated using tangoBlack and veroClear. (b) Hologram 2 fabricated using veroClear. The holes in the holograms were used to attach the two to each other. The 16 holes in (b) allowed for four distinct positions with known displacements. 


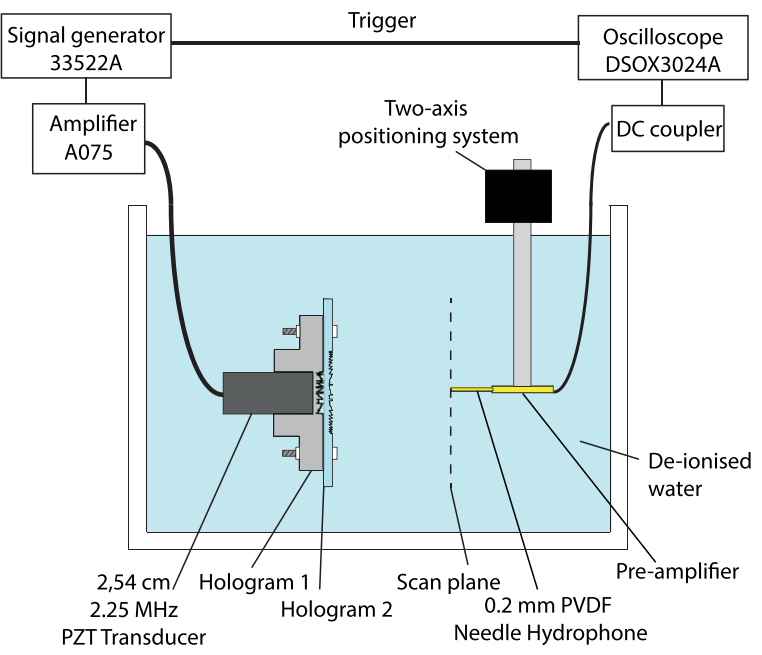

FIG. 5. Schematic of the experimental setup used for characterizing the emitted field.

k-Wave toolbox. ${ }^{20}$ A schematic of the experimental setup can be seen in Fig. 5. Four scans were carried out in total. Between each scan, the second hologram was detached from the first hologram and re-attached in a different position. The relative displacement between each of the four scans was $(0,0),(-5 \mathrm{~mm},-5 \mathrm{~mm}),(5 \mathrm{~mm},-4 \mathrm{~mm}),(0,5 \mathrm{~mm})$.

Figures 6(a)-6(d) show the maximum pressure generated in the target plane for each of the four scans. Both patterns are clearly generated in each dataset, and the translation of the second pattern between each stacking position is clearly visible. This confirms that by reducing the hologram modulation depth and stacking them, it is possible to
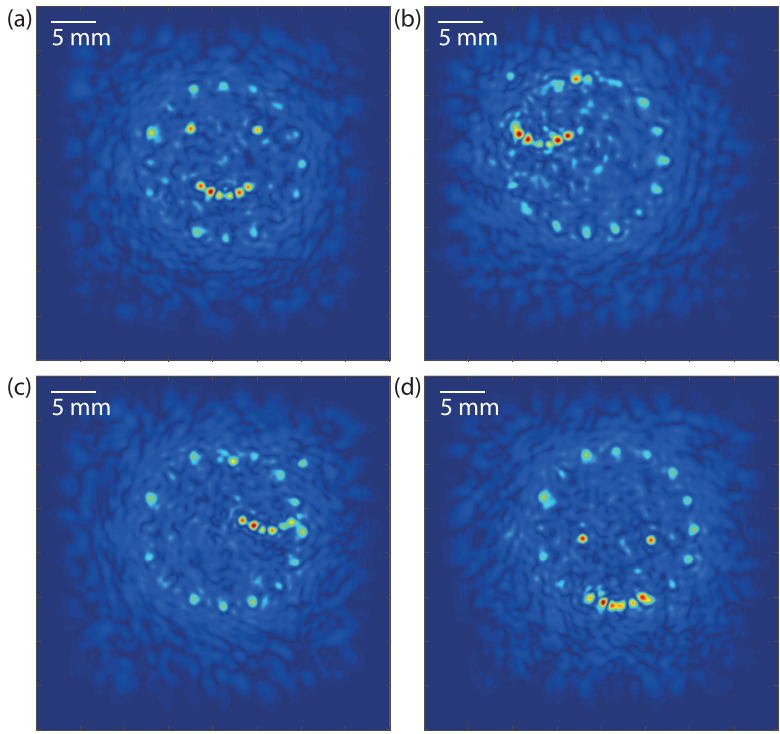

FIG. 6. (a) Maximum pressure at target depth for position 1. (b) Maximum pressure at target depth for position $2(-5 \mathrm{~mm},-5 \mathrm{~mm})$. (c) Maximum pressure at target depth for position $3(5 \mathrm{~mm},-4 \mathrm{~mm})$. (d) Maximum pressure at target depth for position $4(0,5 \mathrm{~mm})$ create acoustic patterns that can be reconfigured by changing the relative position of the individual holograms. One drawback is that the background to the pattern is relatively high. This is because some of the energy is coupled into higher diffraction orders and into the background zeroth order. While these waves are not in focus, they do spatially overlap with the target pattern. In the future, these could be spatially separated from the target patterns by employing an off-axis incident wave as opposed to the normal incidence used here. This approach is used in optical holography. The signal to noise ratio of the two patterns is also different, with the second hologram being higher, due to the different numbers of foci. This could also be optimized in the future by adapting the modulation depth of each hologram to control the relative energy coupled into the $p_{10}$ and $p_{01}$ orders.

To summarize, this work has introduced a technique for creating a complex, reconfigurable, distribution of pressure using a single element transducer via the stacking of independent holograms. This partially overcomes the "static" nature of conventional acoustic holograms which, at present, represents a significant drawback. In the future, this approach could prove valuable to a range of applications for which acoustic holograms are already being applied. For example, in particle/cell trapping and manipulation, the positions of an array of objects might be flexibly reconfigured. ${ }^{21}$ Alternatively, for the acoustic assembly, ${ }^{22}$ the ability to reconfigure the field might enable more complex objects to be formed.

This work was supported by the Engineering and Physical Sciences Research Council. The authors also thank Dr. Eleanor Martin for her assistance with the field scans.

\section{DATA AVAILABILITY}

The data that support the findings of this study are available from the corresponding author upon reasonable request.

\section{REFERENCES}

1A. Marzo, S. Seah, B. Drinkwater, D. Sahoo, B. Long, and S. Subramanian, "Holographic acoustic elements for manipulation of levitated objects," Nat. Commun. 6, 8661 (2015)

${ }^{2}$ G. Memoli, M. Caleap, M. Asakawa, D. Sahoo, B. Drinkwater, and S. Subramanian, "Metamaterial bricks and quantization of meta-surfaces," Nat. Commun. 8, 14608 (2017).

${ }^{3}$ T. Carter, S. Seah, B. Long, B. Drinkwater, and S. Subramanian, "UltraHaptics: Multi-point mid-air haptic feedback for touch surfaces," in UIST 2013: Proceedings of the 26th Annual ACM Symposium on User Interface Software and Technology (2013), p. 505.

${ }^{4}$ R. Hirayama, D. Plasencia, N. Masuda, and S. Subramanian, "A volumetric display for visual, tactile and audio presentation using acoustic trapping," Nature 575, 320 (2019).

${ }^{5}$ Y. Hertzberg and G. Navon, "Bypassing absorbing objects in focused ultrasound using computer generated holographic technique," Med. Phys. 38, 6407 (2011)

${ }^{6}$ G. Maimbourg, A. Houdouin, T. Deffieux, M. Tanter, and J. Aubry, "3Dprinted adaptive acoustic lens as a disruptive technology for transcranial ultrasound therapy using single-element transducers," Phys. Med. Biol. Lett. 63, 025026 (2018).

${ }^{7}$ S. Jiménez-Gambín, N. Jiménez, J. Benlloch, and F. Camarena, "Holograms to focus arbitrary ultrasonic fields through the skull," Phys. Rev. Appl. 12, 014016 (2019).

${ }^{8}$ A. Marzo and B. Drinkwater, "Holographic acoustic tweezers," Proc. Natl. Acad. Sci. 116, 84 (2019).

${ }^{9}$ M. Bakhtiari-Nejad, A. Elnahhas, M. Hajj, and S. Shahab, "Acoustic holograms in contactless ultrasonic power transfer systems: Modeling and experiment," J. Appl. Phys. 124, 244901 (2018). 
${ }^{10}$ P. Kruizinga, P. van der Meulen, A. Fedjajevs, F. Mastik, G. Springeling, N. de Jong, J. Bosch, and G. Leus, "Compressive 3D ultrasound imaging using a single sensor,” Sci. Adv. 3, e1701423 (2017).

${ }^{11} \mathrm{R}$. Lalonde and J. Hunt, "Field conjugate acoustic lenses for ultrasound hyperthermia," IEEE Trans. Ultrason. Ferroelectr. Freq. Control 40, 592 (1993).

${ }^{12}$ K. Melde, A. G. Mark, T. Qiu, and P. Fischer, "Holograms for acoustics," Nature 537, 518 (2016).

${ }^{13}$ J. Zhang, Y. Tian, Y. Cheng, and X. Liu, "Acoustic holography using composite metasurfaces,” Appl. Phys. Lett. 116, 030501 (2020).

${ }^{14}$ L. Cox, K. Melde, A. Croxford, P. Fischer, and B. Drinkwater, "Acoustic hologram enhanced phased arrays for ultrasonic particle manipulation,” Phys. Rev. Appl. 12, 064055 (2019).

${ }^{15} \mathrm{M}$. D. Brown, B. T. Cox, and B. E. Treeby, "Design of multi-frequency kinoforms," Appl. Phys. Lett. 111, 244101 (2017).

${ }^{16} \mathrm{R}$. Lalonde and J. Hunt, "Variable frequency field conjugate lenses for ultrasound hyperthermia," IEEE Trans. Ultrason. Ferroelectr. Freq. Control 42, 825 (1995).
${ }^{17}$ M. Norasikin, D. Martinez Plasencia, S. Polychronopoulos, G. Memoli, Y. Tokuda, and S. Subramanian, "SoundBender: Dynamic acoustic control behind obstacles," in the 31st Annual ACM Symposium on User Interface Software and Technology (ACM, Berlin, Germany, 2018), p. 247.

${ }^{18}$ V. Moreno, J. Roman, and J. Salgueiro, "High efficiency diffractive lenses: Deduction of kinoform profile,” Am. J. Phys. 65, 556 (1997).

${ }^{19} \mathrm{~S}$. Mellin and G. Nordin, "Limits of scalar diffraction theory and an iterative angular spectrum algorithm for finite aperture diffractive optical element design," Opt. Express 8, 705 (2001).

${ }^{20} \mathrm{~B}$. Treeby and B. Cox, "k-Wave: MATLAB toolbox for the simulation and reconstruction of photoacoustic wave-fields," J. Biomed. Opt. 15, 021314 (2010).

${ }^{21}$ Z. Ma, A. Holle, K. Melde, T. Qiu, K. Poeppel, V. Kadiri, and P. Fischer, "Acoustic holographic cell patterning in a biocompatible hydrogel," Adv. Mater. 32, 1904181 (2020).

${ }^{22}$ K. Melde, E. Choi, Z. Wu, S. Palagi, T. Qiu, and P. Fischer, "Acoustic fabrication via the assembly and fusion of particles," Adv. Mater. 30, 1704507 (2018). 